\title{
Skepticism and Epistemic Closure: Two Bayesian Accounts
}

\author{
Luca Moretti \\ University of Aberdeen \\ Munich Center for Mathematical Philosophy \\ 1.moretti@abdn.ac.uk
}

\author{
Tomoji Shogenji \\ Rhode Island College \\ TShogenji@ric.edu
}

\begin{abstract}
This paper considers two novel Bayesian responses to a well-known skeptical paradox. The paradox consists of three intuitions: first, given appropriate sense experience, we have justification for accepting the relevant proposition about the external world; second, we have justification for expanding the body of accepted propositions through known entailment; third, we do not have justification for accepting that we are not disembodied souls in an immaterial world deceived by an evil demon. The first response we consider rejects the third intuition and proposes an explanation of why we have a faulty intuition. The second response, which we favor, accommodates all three intuitions; it reconciles the first and the third intuition by the dual component model of justification, and defends the second intuition by distinguishing two principles of epistemic closure.
\end{abstract}

\section{Keywords}

skepticism; epistemic closure; Bayesianism; epistemic justification; Linda case

\section{Introduction}

In this paper we consider two Bayesian responses to a well-known skeptical paradox that arises from the principle of epistemic closure under known entailment. We assume that perceptual evidence is propositional $^{1}$ and we formulate an instance of the paradox as follows:

$e$ : My experience is that of a hand held up in front of my face.

So,

$h$ : Here is a hand in front of my face.

So,

$\neg d$ : I am not a disembodied soul in an immaterial world deceived by an evil demon into seeing a hand image.

\footnotetext{
${ }^{1}$ For a defence of propositional evidence see for instance Williamson (2000).
} 
The paradox consists of three intuitions. The first is that once we obtain $e$, we have justification for accepting $h$, and more generally we have justification for accepting many particular propositions about the external world when we have appropriate sense experience. ${ }^{2}$ The second intuition is that we have justification for expanding the body of accepted propositions through known entailment. It follows from these two intuitions, apparently, that once we obtain $e$, we have justification for accepting $\neg d$ since we have justification for accepting $h$ and we know that $h$ entails $\neg d$. However, we have the third intuition that we do not have justification for accepting $\neg d$ given $e$; that is, it seems unreasonable to reject $d$ since $e$ is exactly the kind of experience we expect if $d$ is true.

There are three obvious reactions to this paradox. First, we may give up the intuition that once we obtain $e$, we have justification for accepting $h$. The reasoning for this is Modus Tollens: if we have justification for accepting $h$ given $e$, then via epistemic closure we have justification for accepting $\neg d$ given $e$. But we do not have justification for accepting $\neg d$ given $e$. So, we do not have justification for accepting $h$ when $e$ is given to us. This way out looks rather unattractive: since $h$ can be replaced by any proposition about the external world with $e$ and $d$ adjusted accordingly, the result is the unpalatable position of global skepticism.

Second, we may give up the intuition that we have justification for expanding the body of accepted propositions through known entailment. This makes it possible to maintain that we have justification for accepting $h$, while conceding that we do not have justification for accepting $\neg d$. We can avert global skepticism in this way, but it would be

${ }^{2}$ Our interest throughout this paper is propositional justification. So, we do not address the issue of the basing relation. We say "we have justification for accepting $p$ " without implying that we actually accept $p$, let alone we accept $p$ for the right reason. We will explain shortly why we formulate the paradox in terms of acceptance instead of belief. 
cognitively devastating if we could not use known entailment to expand the body of accepted propositions.

Third, we may stand by the first two intuitions and conclude by Modus Ponens that once we obtain $e$, we have justification for accepting $\neg d$. Some of the currently popular positions on skepticism defend this claim by rejecting the terms of debate in which the skeptical challenge is traditionally developed. The externalists reject the requirement that the grounds of justification must be available to the subject. The internalists want to retain this requirement, but many of them move towards externalism in abandoning the further requirement that the adequacy of the grounds must be established by evidence available to the subject. Changing the terms of debate allows those epistemologists to undercut the skeptical challenge.

Here we cannot fully assess whether it is reasonable to change the terms of debate in this way. We only make two brief remarks. First, there is a serious concern that changing the terms of debate makes it too easy to acquire justification for accepting $\neg d{ }^{3}$ Assume for the sake of argument that $d$ is true: I am actually a disembodied soul in an immaterial world deceived by an evil demon into seeing a hand image. The assumption renders $e$ useless as grounds for accepting $h$, so that $e$ is no longer adequate grounds for accepting $h$ 's logical consequence $\neg d$. In light of this when we use $e$ as grounds for accepting $\neg d$, we are relying on $e$ to eliminate the possibility that $e$ itself is useless as grounds for accepting $\neg d$. This seems intuitively incorrect (in the same way it is incorrect

\footnotetext{
${ }^{3}$ This line of argument was originally brought up against externalism (Fumerton 1995, pp. 177-180; Vogel 2000), but it was soon pointed out that the same problem arises for those versions of internalism that lean towards externalism in not requiring the adequacy of the grounds for justification to be established by evidence available to the subject (Cohen 2002, van Cleve 2003). It is customary to formulate the problem against accounts of knowledge, but it applies to accounts of justification as well (cf. Vogel 2008).
} 
to rely on the witness's self-report to answer questions about the report's reliability). If so, then we cannot obtain justification for accepting $\neg d$ by simply acquiring $e$.

Our second remark on changing the terms of debate concerns the extent of its effectiveness. The externalist (or externalist-leaning) conception of justification may be appropriate in regard to belief, which is arguably a disposition and whose formation may not require conscious decision. The externalist can maintain with some plausibility that we are justified in having a favourable disposition toward $\neg d$ even if we do not have good reason for accepting $\neg d$, provided the disposition was formed properly. However, the externalist conception is much less convincing as an account of justification for the conscious act of acceptance. ${ }^{4}$ If we do not have good reason for accepting $\neg d$, then we do not have justification for accepting it even if the conditions we are unaware of are favorable. This means that the skeptical paradox remains a threat in regard to acceptance even if we can escape it for beliefs by changing the terms of debate. ${ }^{5}$ It is for this reason that we formulate the skeptical paradox in regard to acceptance instead of belief.

We consider two novel Bayesian responses to the paradox. The first response (Section 2) draws on the idea of transmission failure, viz. justification that $e$ provides for $h$ fails to transmit to $\neg d$. Transmission failure explains why we are unwilling to embrace the reasoning from $e$ to $\neg d$ through $h$. The first response we consider proceeds further to explain not only the intuition against the reasoning, but the intuition against $\neg d$ itself, viz. we can regard it as an instance of a pervasive fallacy in the assessment of probabilities. One attraction of this response is that it keeps the familiar Bayesian model of justification

\footnotetext{
${ }^{4}$ See Cohen (1992) and Alston (1996) for the distinction of belief and acceptance in epistemology. We will say a little more about the notion of acceptance in Section 3. ${ }^{5}$ Those who favor the traditional terms of debate on skepticism in regard to belief may substitute "belief" for "acceptance" throughout the paper.
} 
intact in which justification is closed under entailment. However, despite these features, we do not endorse this response in the end. One reason is that its success hinges on the problematic claim that we have a priori justification for accepting $\neg d$. Another reason is that it merely explains away the third intuition against $\neg d$, while the second response takes all three intuitions at face value and accommodates them.

The second response, which we endorse, reconciles the first and the third intuition by abandoning the familiar Bayesian model of justification in favor of a new model that has its roots in philosophy of science, which we call the dual component model of justification (Sections 3 and 4). Since the dual component model of justification allows that we have justification for accepting $h$ but not for accepting $\neg d$, the usual form of epistemic closure does not hold in the model. However, this does not mean that we cannot expand the body of accepted propositions through known entailment. We show that a weaker form of epistemic closure holds in the model and is sufficient for the expansion (Section 5).

To clarify the nature of the second response, it does not try to establish the correctness of the three intuitions. In particular, we do not try to establish that we have justification for accepting $h$ when given $e$. Its limited goal is to show that the three intuitions are compatible, and thus defending the first two intuitions does not force us to insist that we have justification for accepting $\neg d$. We believe it is important to recognize this option since there are many epistemologists who find all three intuitions compelling. 


\section{The skeptical paradox, transmission failure, and the conjunction fallacy}

We begin with an explication of epistemic justification familiar to the Bayesians. The explication is based on what Richard Foley (2009) dubbed the Lockean thesis, according to which we rationally believe $x$ just in case $P(x) \geq t$, where $P(x)$ is a rational degree of confidence in $x$ and $t$ is some threshold value of sufficiency. Many epistemologists embrace the Lockean view about justification of belief, viz. we have justification for believing $x$ just in case $P(x) \geq t$. To be more precise, the relevant probability is the probability assigned on the basis of evidence $y$, so that we have justification for believing $x$ given $y$ just in case $P_{y}(x) \geq t$ (where $P_{y}$ is our probability function updated on the acquisition of evidence $y$. We use the standard Bayesian updating rule that the posterior probability $P_{y}(x)$ should equal the prior conditional probability $P(x \mid y) .{ }^{6} \mathrm{We}$ shall call it "the Lockean model" of justification of belief.

The Lockean model assumes that belief is a discrete concept rather than a gradual one-either we believe $x$ or not believe $x$ - and that we have sufficient justification for believing $x$ when the degree of justification reaches the threshold value. This assumption is not uncontroversial since we can speak sensibly of stronger and weaker belief. One could make the case that belief is fundamentally a gradual concept just as being tall is, and that the apparently non-gradual use of "belief" is implicitly comparative in the same way the apparently non-gradual use of "tall" is implicitly comparative. We need not resolve this dispute here since we formulate the skeptical paradox in terms of acceptance instead of belief; it is clear that acceptance is a discrete concept. Hereafter, the term "the

\footnotetext{
${ }^{6}$ To be even more precise, the probability assignment is relative to the background information $b$, so that we have justification for believing $x$ just in case $P_{y \wedge b}(x) \geq t$. We take the background information $b$ to be empty in the discussion of the skeptical challenge.
} 
Lockean model" refers to the view that we have justification for accepting $x$ given $y$ just in case $P_{y}(x) \geq t$.

It is well known that the Lockean model does not support multi-premise closure, i.e. $P_{y}\left(x_{1}\right), \ldots, P_{y}\left(x_{n}\right) \geq t$ and $\left\{x_{1}, \ldots x_{n}\right\}-x_{n+1}$ together do not entail $P_{y}\left(x_{n+1}\right) \geq t$. But the type of closure that is relevant to the skeptical paradox and which the Lockean model does support is single-premise closure. It is a theorem of the probability calculus that if $P_{y}\left(x_{1}\right) \geq t$ and $x_{1} \vdash x_{2}$, then $P_{y}\left(x_{2}\right) \geq t$. There is therefore no way that $P_{e}(h) \geq t$ while $P_{e}(\neg d)<t$ in the Lockean model since $h$ entails $\neg d$. So, if we have justification for accepting $h$ given $e$, we also have justification for accepting $\neg d$ given $e$.

In light of this consequence, some epistemologists who subscribe to the Lockean model but question the reasoning in support of $\neg d$ turned their attention to the transmission of acquired justification. ${ }^{7}$ Let us grant here that when given $e$, we have justification for accepting $h$, and hence for accepting $\neg d$ by epistemic closure. This does not mean, they point out, that we acquire justification for $\neg d$ from $e$ (through $h$ by entailment). Having justification given $e$ is not the same as acquiring justification from $e$. Those epistemologists propose that we have justification for accepting $\neg d$ regardless of $e$, that is, prior to and independently of the acquisition of $e$. Indeed, once we deny that $\neg d$ receives justification from $e$, the only way to block the Modus Tollens argument against justification of $h$ is to maintain that we have justification for accepting $\neg d$ independently of $e$. Thus, Crispin Wright $(2002 ; 2007 ; 2014)$ contends that we have justification for accepting $h$ given $e$ only if we have prior and independent justification for accepting

\footnotetext{
${ }^{7}$ For a general introduction to the topics of transmission and failure of transmission of justification see Moretti and Piazza (2013).
} 
"cornerstone" propositions like $\neg d$. Wright's view $(2004 ; 2007 ; 2014)$ is that we are $a$ priori entitled to accept cornerstone propositions of this type.

The account raises the hope of resolving the skeptical paradox. If justification for $\neg d$ does not come from $e$, then we need not defend the odd claim that $d$ is refuted by the evidence $e$ that is entailed by $d$. So, it does not face the problem of "easy justification". The account also explains our unwillingness to embrace the reasoning from $e$ to $\neg d$ through $h$ because justification for accepting $\neg d$ does not come from $e$. Of course, the third intuition is not about the reasoning, but the result of the reasoning-that we have justification for accepting $\neg d$. The novelty of the first response to the skeptical paradox we consider is its explanation of why we have a strong intuition against this claim.

The explanation begins with a formal analysis of transmission failure. It is noted in the course of the debate on skepticism and transmission failure that a formal model appropriate for an acquisition of justification is that of incremental confirmation (cf. Okasha 2004 and White 2006). We acquire justification for $\neg d$ from $e$ only if there is an increase in the probability of $\neg d$ by $e$, or $P_{e}(\neg d)>P(\neg d)$. This is different from having justification as explicated by the Lockean model. We can have justification for accepting $\neg d$ given $e$ even if we acquire no justification for accepting $\neg d$ from $e$ because $P_{e}(\neg d) \geq t$ is compatible with $P_{e}(\neg d) \leq P(\neg d)$. An interesting part of this analysis is that $P_{e}(h)>P(h)$ and $h \vdash \neg d$ together do not entail $P_{e}(\neg d)>P(\neg d)$, and thus even if we acquire justification for $h$ from $e$, and $h$ entails $\neg d$, we may not acquire justification for $\neg d$ from $e$. The formal interpretation supports the view that acquired justification is not always transmitted through known entailment. 
The case for transmission failure has been made most convincingly in probabilistic terms by White (2006). As mentioned already, $e$ is exactly the kind of experience we expect if we are deceived by an evil demon into seeing a hand image. So, $d$ makes $e$ more likely, i.e. $P(e \mid d)>P(e)$. It follows by Bayes' Theorem and the standard Bayesian updating that $P_{e}(d)>P(d),{ }^{8}$ and thus $P_{e}(\neg d)<P(\neg d)$. This means that $\neg d$ is disconfirmed by $e$. So, even though $P_{e}(\neg d) \geq t$ follows from $P_{e}(h) \geq t$ and $h \models \neg d$, no part of justification for $\neg d$ is acquired from $e$. This combination-that we have justification for accepting $\neg d$ given $e$, but $\neg d$ is disconfirmed by $e$-opens a way of explaining the intuition that we do not have justification for accepting $\neg d$; viz. we can regard it as an instance of a pervasive fallacy in the assessment of probabilities uncovered by the "heuristics and biases" research in psychology.

Let us see how the pervasive fallacy occurs in the widely discussed case of Linda (Tversky and Kahneman 1983). The participants in the experiment are asked which of the two propositions, $p$ and its logical consequence $q$, is more probable given information $i$ :

$i$ : Linda is 31 years old, single, outspoken, and very bright. She majored in philosophy. As a student, she was deeply concerned with issues of discrimination and social justice, and also participated in anti-nuclear demonstrations.

$p$ : Linda is a bank teller and is active in the feminist movement.

$q$ : Linda is a bank teller.

To the dismay of logicians many participants answer that $p$ is more probable than $q$ thereby committing "the conjunction fallacy," i.e. the fallacy of assigning a higher

\footnotetext{
${ }^{8}$ By Bayes theorem $P(e \mid d)=P(d \mid e) P(e) / P(d)$, hence $P(e \mid d) / P(e)=P(d \mid e) / P(d)$. So, it follows from $P(e \mid d)>P(e)$ that $P(d \mid e)>P(d)$. This entails, via the standard Bayesian updating, that $P_{e}(d)>P(d)$.
} 
probability to the conjunction than to one of its conjuncts. It is impossible that $P_{i}(p)>$ $P_{i}(q)$ since the conjunction $p$ entails the conjunct $q$.

The Linda case is not an isolated phenomenon. Time and again many people commit the conjunction fallacy in similar cases. One influential formal analysis of the conjunction fallacy is that the fallacy occurs when the incremental confirmation of the conjunct is greater than that of the conjunction (Sides et al. 2002; Crupi et al. 2008). To apply the analysis to the Linda case, the information $i$ confirms the conjunction $p$ in the incremental sense of $P_{i}(p)>P(p)$ because we acquire some justification for believing $p$ from $i$. Meanwhile, $i$ disconfirms the conjunct $q$ in the sense of $P_{i}(q)<P(q)$ because we acquire some justification for accepting the negation $\neg q$ from $i$. Many participants in the experiment confuse an increase in probability with a high probability and answer incorrectly that $p$ is more probable than $q$ given $i$, according to the confirmation analysis.

The conjunction fallacy and its confirmation analysis suggest that we routinely confuse an increase in probability with a high probability, and a decrease in probability with a low probability. If we apply this analysis to the skeptical paradox, we should expect many people assign a high probability to $d$ given $e$ and a low probability to $\neg d$ given $e$ since $e$ confirms $d$ and disconfirms $\neg d$, even though it is inconsistent with a high probability assigned to $h$. As a result, we have the faulty intuition that we do not have justification for accepting $\neg d$ given $e$. It is not an ad hoc account since the confusion responsible for the faulty intuition is commonplace.

In sum, the first response to the skeptical paradox combines the Lockean model of justification with the Bayesian account of a pervasive fallacy in the assessment of probabilities. The Lockean model makes the three intuitions incompatible, but the 
Bayesian account of the fallacy explains why we have the faulty third intuition. If the analysis is correct, then the skeptic is exploiting the pervasive fallacy in the assessment of probabilities to induce doubt about the external world.

Although this response to the skeptical paradox has considerable merits and it might work at the end, we also have strong reservations. The weakest part of the response is its account of justification for accepting $\neg d$. It does not come from $e$. We cannot claim it comes from some other sense experience since the skeptic can easily adjust the demon hypothesis so that the sense experience in question confirms the demon hypothesis. So, justification for accepting $\neg d$ is a priori. Note also that it follows from $P_{e}(h) \leq P_{e}(\neg d)$ and $P_{e}(\neg d)<P(\neg d)$ that $P_{e}(h)<P(\neg d)$. As a result, we need a priori justification for $\neg d$ in order to have empirical justification for $h$, i.e. $P_{e}(h) \geq t$ only if $P(\neg d) \geq t$. Both claims - that we have a priori justification for accepting $\neg d$ and that we need a priori justification for $\neg d$ for empirical justification for $h$ —appear highly questionable. ${ }^{9}$

We may tolerate questionable consequences in the absence of strong alternatives, but the second response to the skeptical paradox we consider in the next three sections does provide a strong alternative - it takes all three intuitions at face value and accommodates them, instead of explaining away one of the intuitions.

\footnotetext{
${ }^{9}$ For instance the adequacy of White's (2006) formal assumptions and thus the actual epistemological bearing of his results have been questioned by Weatherson (2007), Kung (2010) and Pryor (2013). Furthermore Jenkins (2007) and Pritchard (2005b), among others, have argued that Wright's entitlement is at the very best a pragmatic state that cannot epistemically justify belief acceptance.
} 


\section{The dual component model of justification}

The second response to the skeptical paradox has two parts. First, it reconciles the first and the third intuition by replacing the Lockean model of justification with a new model, which we call the dual component model. This section introduces the new model and the next section explains the way it reconciles the first and the third intuition. The second part is an account of epistemic closure. The customary form of epistemic closure does not hold in the dual component model, but we show in Section 5 that a weaker form of epistemic closure holds in the model and is sufficient for expanding the body of accepted propositions through known entailment.

Before presenting the new model of justification, we note that a well-known informal account of knowledge that denies closure is of no help for our purpose. Dretske (2005) rejects the thesis that knowledge is closed under known entailment, stating that if a subject $S$ knows $h, S$ ought also to know $h$ 's lightweight logical consequences but not necessarily $h$ 's heavyweight (or limiting) logical consequences, such as $\neg d$. This would follow from the account of knowledge first provided by Dretske (1971), ${ }^{10}$ according to which $S$ knows $p$ just in case $S$ has a conclusive reason $R$ for $p$, where $R$ is a conclusive reason for $p$ if and only if (1) $S$ is aware that $R$ is true, and (2) $R$ would not be true if $p$ were not true. It is important in his account that $S$ can possess a conclusive reason $R$ for $p$ even if $S$ cannot recognize (even in principle) that (2) is satisfied. In the case of the skeptical paradox, since $S$ may possess a conclusive reason for $h$ without having any conclusive reason for its consequence $\neg d, S$ may know $h$ without knowing $\neg d$.

One might think of modifying Dretske's notion of conclusive reason as a notion about epistemic justification. In other words, $S$ has justification for accepting $p$ just in

${ }^{10}$ His account of knowledge is modified and supplemented by Nozick (1981). 
case $S$ has a conclusive reason $R$ for $p$. Since $S$ may possess a conclusive reason for $h$ without having conclusive reasons for $\neg d, S$ may have justification for accepting $h$ without having justification for accepting $\neg d$. So, epistemic justification is not closed under known entailment. But this modification of Dretske's analysis is quite problematic, for it entails that $S$ cannot have any justification for accepting any proposition $p$ if $p$ is false. This seems incorrect, though its counterpart in knowledge- $S$ cannot know any proposition $p$ if $p$ is false-seems correct.

Suppose, however, we decide to disregard this counterintuitive consequence. There still remains a problem. Note that this notion of justification is not wholly internalist, in that it depends in part on features of propositions that may not be cognitively accessible to the epistemic subject. For this reason, Dretske has often been criticized for changing the terms of debate on skepticism, which seem to require that any factor necessary to epistemically justify or make the subject know must be cognitively accessible to the subject. We do not take side on this issue. As we noted earlier, the externalist (or externalist-leaning) conception of justification may be appropriate in regard to belief, and this may extend to knowledge, but it is not plausible as an account of justification for acceptance, where the skeptical paradox remains a threat even if we can escape it for beliefs by changing the terms of debate. In contrast the dual component model of justification introduced in this section is applicable to acceptance, where the concept of justification is wholly internalist.

We now describe the dual component model of justification. As noted already, since $h$ entails $\neg d, \neg d$ is at least as probable as $h$. So, how can we have justification for accepting $h$ but not $\neg d$ ? According to the dual component model of justification, 
cognition has two goals. We want propositions we accept to be true rather than false, so it is better for the proposition we accept to be more probable. That reduces the risk of accepting a false proposition. However, we also want the proposition we accept to be informative. A cognitively valuable proposition must be both of low risk and informative. These two cognitive goals - of low risk and informative- often pull us in the opposite directions. This is a familiar theme in philosophy of science:

Science does not aim, primarily, at high probabilities. It aims at a high informative content, well backed by experience. But a hypothesis may be very probable simply because it tells us nothing, or very little. A high degree of probability is therefore not an indication of 'goodness' - it may be merely a symptom of low informative content. (Popper 1954, p.146, original Italics.)

In our view the point applies not just to the evaluation of a scientific hypothesis but to the evaluation of any proposition for acceptance. We can make propositions we accept more informative by making them more specific, but more specific propositions are more likely to be false, other things being equal, and thus riskier.

The standard measure of information, $I(x)=-\log P(x)=\log [1 / P(x)]$, bears this out. For example, $x$ carries $1,2,3, \ldots$ bits of information when $P(x)=1 / 2,1 / 4,1 / 8, \ldots$, respectively, since $\log _{2} 2=1, \log _{2} 4=2, \log _{2} 8=3, \ldots$ The amount of information $I(x)$ and the probability $P(x)$ are inversely related. ${ }^{11}$ Ideally, the proposition we accept should be highly informative and thus highly improbable a priori, while the evidence we possess should show it to be very probably true. In this case we have strong justification for accepting the proposition. On the other hand, when the evidence leaves room for some

${ }^{11}$ The standard measure of information is due to Shannon and Weaver (1949). Two straightforward alternatives are $I(x)=1-P(x)$ and $I(x)=1 / P(x)$. The former is used, among others, in Bar-Hillel and Carnap (1953) and Popper (1963, p. 392). The latter is mentioned in Levi (2004, Section 4.2). The choice of a specific measure does not affect the point that the amount of information and the probability are inversely related. 
doubt, the dual component model suggests we balance risk and informativeness, just as we consider both the probability and the payoff in making a wager. ${ }^{12}$ As a result, we sometimes have stronger justification for accepting the less probable of two propositions. The dual component model is taken seriously in philosophy of science, ${ }^{13}$ but in our view the model is applicable to epistemic evaluation in general.

Some may grant that informativeness is a desirable feature of a proposition but question the idea that it is epistemically desirable. It may be suggested, for example, that informativeness is a non-epistemic component in the evaluation of a proposition comparable to utility in decision theory that must be combined with the epistemic component. Some might suggest further that there should be no trade-off between the epistemic and the non-epistemic component of evaluation since we cannot accept a proposition whose (posterior) probability is not sufficiently high no matter how informative it is. In other words, epistemic justification in the Lockean sense of $P_{e}(h) \geq t$ is a necessary condition for accepting $h$ given $e$. Of these two suggestions the first is to some extent a matter of judgment about the natural way of parsing different aspects of evaluation and what to call the different aspects; while the second suggestion against trade-off raises a substantive issue of how the components of evaluation relate to each other. So, we address the second suggestion first.

Our position is that we cannot draw a threshold of probability to set the limit of risk as in the Lockean model since informativeness and risk are intertwined in the evaluation of a proposition for acceptance. We want to illustrate this point with a simple

\footnotetext{
12 See Levi $(1967 ; 1980)$ for an implementation of this idea.

${ }^{13}$ See Huber (2008a, 2008b) for recent work on the dual component model in philosophy of science.
} 
example. Consider two propositions $h_{1}$ and $h_{2}$ that are equally informative and equally risky to accept. Under these conditions, we have justification for accepting $h_{1}$ just in case we have justification for accepting $h_{2}$ both by the Lockean model and by the dual component model since the two propositions share the same profile in all relevant aspects. Assume further that the two propositions are irrelevant to each other, so that accepting one of them does not affect the evaluation of the other. In a natural understanding of justification this means that if we have justification for accepting $h_{1}$, then we have justification for accepting the conjunction of $h_{1}$ and $h_{2}$ by the following reasoning. We have justification for accepting $h_{1}$ by hypothesis. It follows that we also have justification for accepting $h_{2}$. But accepting $h_{1}$ does not affect the evaluation of $h_{2}$. So, we have justification for accepting $h_{1}$ first, and then $h_{2}$ subsequently. This means that we have justification for accepting $h_{1} \wedge h_{2}{ }^{14}$

Note that this understanding of justification is inconsistent with the Lockean model that sets the limit of risk by a threshold of probability. Since the conjunction $h_{1} \wedge$ $h_{2}$ is less probable than the conjunct $h_{1}$ in this case, it is possible by the Lockean model that we have justification for accepting $h_{1}$ but not for accepting $h_{1} \wedge h_{2}{ }^{15}$ In contrast, the dual component model can explain why we have justification for accepting $h_{1} \wedge h_{2}$ in this case, viz. the greater risk of accepting $h_{1} \wedge h_{2}$ is counterbalanced by the greater amount of information $h_{1} \wedge h_{2}$ carries. Although this is an artificial example for the purpose of illustration, the general point applies to cases we come across in real life; for example, it

\footnotetext{
${ }^{14}$ This is a special case of multiple-premise closure, where the two premises are irrelevant to each other and the conclusion is the conjunction of the premises. ${ }^{15}$ Unless, of course, we demand Cartesian certainty for justification by setting the threshold $t$ of the Lockean model at 1. It would then follow trivially from $P_{e}\left(h_{1}\right) \geq 1$ and $P_{e}\left(h_{2}\right) \geq 1$ that $P_{e}\left(h_{1} \wedge h_{2}\right) \geq 1$.
} 
is for the same reason of tolerating a greater risk for a greater amount of information that we often have justification for accepting a large body of propositions contained in a textbook even if the probability is low that all of the propositions are true. ${ }^{16}$

We now address the first suggestion that informativeness is a non-epistemic component comparable to utility in the standard decision theory. As stated already, what to call different aspects of evaluation is to some extent a matter of judgment. We would not insist on our terminology as long as the substance of our response to the skeptical paradox is understood and endorsed, but we want to make some remarks in favor of informativeness as an epistemic component since they have bearing on the controversial claim of pragmatic encroachment in epistemology (Fantl \& McGrath 2002; 2007; 2009; 2012). First, a proposition that is more informative in the sense used in the dual component model is a proposition with a lower prior probability. It does not mean that it provides information that is useful for attaining some practical objective we are interested in. On the contrary, informativeness in the relevant sense is a central component of the epistemic project of gaining more truth (more information that is true) while avoiding falsity as much as possible, independently of its practical application. Some might point out that we accept propositions so that we can rely on them in the future when we decide on an action to take for attaining some practical objective. However, we cannot take account of utilities in the absence of a specific practical project. When we evaluate a proposition for acceptance with no immediate practical objective to attain, and thus the evaluation is entirely epistemic, we can only consider epistemic features of a proposition, which are risk and informativeness.

\footnotetext{
${ }^{16}$ In this way the duel component model provides a natural solution to Makinson's (1965) preface paradox.
} 
To clarify our stance, we do not deny that the role of informativeness in the evaluation of a proposition for acceptance may be comparable to that of utility in the standard decision theory, but that is not a good reason for thinking that informativeness is a non-epistemic feature. When we evaluate the practical value of a possible action, we need to consider its utilities under different scenarios as well as the probabilities of those scenarios, where only the assessment of the probabilities is epistemic. As a result, when we decide to take an action appropriate under one scenario but not others, it looks as though we have accepted the scenario as true based in part on pragmatic considerations. However, as we use the term in this paper, "accepting a proposition" means that we incorporate it into our stable body of propositions that form the basis of our deliberation. When we evaluate the epistemic value of a proposition for acceptance in this sense, we consider the informativeness of the proposition, instead of the utility of an action under the condition that the proposition is true. It is only for evaluating the practical value of an action that we combine the epistemic and the non-epistemic components. The two components we combine are both epistemic when we evaluate a proposition for acceptance.

Though we cannot take up the issue in this paper, we need to be particularly cautious when we handle cases where epistemic considerations are subservient to the evaluation of a possible action, as is common in the discussion of pragmatic encroachment in epistemology. They may lead us to the skewed picture that pragmatic considerations are an integral part of epistemic evaluation, or if we reject the skewed picture, then to the partial picture that only (posterior) probabilities matter in the epistemic evaluation of a proposition. In a fully epistemic evaluation of a proposition for 
acceptance that is not subservient to the evaluation of a possible action, there are two features of a proposition to consider, which are risk and informativeness, and both of them are epistemic features of a proposition. ${ }^{17}$

\section{Applications of the dual component model}

Having made a case for the dual component model of justification, we now see how the model works in the cases we discussed earlier. In order to see how the model works, it is helpful to have a formal definition of justification that exemplifies the model. We adopt the standard measurer $-\log P(x)=\log [1 / P(x)]$ for informativeness. To match its logarithmic form, we measure the risk of believing a false proposition by $-\log P_{y}(x)=$ $\log \left[1 / P_{y}(x)\right]$, which is inversely related to the posterior probability $P_{y}(x) .{ }^{18}$ What counts for justification—we submit—is not the amount of risk $\log \left[1 / P_{y}(x)\right]$ per se, but the ratio of the amount of risk to the amount of information, $\log \left[1 / P_{y}(x)\right] / \log [1 / P(x)]$. As the risk ratio for the proposition goes down-due to the lower amount of risk or the greater amount of information - the epistemic value of the proposition goes up. So, we adopt the following as measure of the epistemic value: ${ }^{19}$

${ }^{17}$ This may explain why the dual component model of justification is taken more seriously in philosophy of science. It is uncommon in scientific investigation that the evaluation of a hypothesis is subservient to the evaluation of an action to take for attaining a practical objective.

${ }^{18}$ The deeper reason for measuring the risk by $\log \left[1 / P_{y}(x)\right]$ instead of $1-P_{y}(x)$ is the case described in the preceding section, where two propositions $h_{1}$ and $h_{2}$ are equally informative, equally risky to accept, and irrelevant to each other. We showed there that if we have justification for accepting $h_{1}$, then we have justification for accepting the conjunction of $h_{1}$ and $h_{2}$, and that we need to replace the Lockean model by the dual component model to allow this inference. It turns out to be necessary for technical reasons (Shogenji 2012; Atkinson 2012) that we measure the risk by $\log \left[1 / P_{y}(x)\right]$ instead of $1-P_{y}(x)$ to allow the inference. See also footnote 20 below.

${ }^{19}$ It is not necessary to adopt this particular measure for the dual component model as long as the measure is inversely related to $\log [1 / \mathrm{Pe}(\mathrm{x})] / \log [1 / \mathrm{P}(\mathrm{x})]$. Any such alternative 


$$
J(x, y)=1 \frac{\log \left[1 / P_{y}(x)\right]}{\log [1 / P(x)]}=\frac{\log P_{y}(x) \log P(x)}{\log P(x)}
$$

When the epistemic value of $x$ is sufficiently high (i.e. when the risk ratio is sufficiently low), we have justification for accepting $x$. So, the following is a formal definition of justification in the dual component model: given evidence $y$, we have justification for accepting proposition $x$ just in case $J(x, y) \geq t$, where $t$ is some threshold value of sufficiency. It is easy to verify that the epistemic value of $h_{1} \wedge h_{2}$ given $e$ is the same as the epidemic value of $h_{1}$ given $e$ in the example above where $h_{1}$ and $h_{2}$ share the same probabilistic profile and are mutually irrelevant to each other, ${ }^{20}$ so that if we have justification for accepting $h_{1}$ given $e$, then we have justification for accepting $h_{1} \wedge h_{2}$ given $e$.

There is a special case we need to treat separately, viz. a case in which $P(x)=1$, for example when $x$ is a known logical truth. It is intuitively clear that we have justification for accepting $x$ in such a case. However, when $P(x)=1$, the epistemic value $J(x, y)=1-\log \left[1 / P_{y}(x)\right] / \log [1 / P(x)]$ is undefined. We can solve this problem by

measure $J^{*}$ is ordinally equivalent to $J$ in the sense that for any two pairs $\left\langle x_{1}, y_{1}\right\rangle$ and $<x_{2}$, $y_{2}>, J\left(x_{1}, y_{1}\right)>/=/<J\left(x_{2}, y_{2}\right)$ if and only if $J^{*}\left(x_{1}, y_{1}\right)>/=/<J^{*}\left(x_{2}, y_{2}\right)$ (Shogenji 2012; Atkinson 2012). When necessary, we relativize the prior and posterior probabilities to the background information $z$, so that the prior probability is $P_{z}(x)$ while the posterior probability is $P_{z \wedge y}(x)$.

${ }^{20}$ The risk ratio for $h_{1} \wedge h_{2}$ is $\log \left[1 / P_{e}\left(h_{1} \wedge h_{2}\right)\right] / \log \left[1 / P\left(P\left(h_{1} \wedge h_{2}\right)\right]\right.$. By the hypothesis of mutual irrelevance (probabilistic independence), this amounts to $\log \left[1 / P_{e}\left(h_{1}\right) \times\right.$ $\left.1 / P_{e}\left(h_{2}\right)\right] / \log \left[1 / P\left(h_{1}\right) \times 1 / P\left(h_{2}\right)\right]=\left(\log \left[1 / P_{e}\left(h_{1}\right)\right]+\log \left[1 / P_{e}\left(h_{2}\right)\right]\right) /\left(\log \left[1 / P\left(h_{1}\right)\right]+\log \right.$ $\left.\left[1 / P\left(h_{2}\right)\right]\right)$. This ratio is the same as the risk ratio for $h_{1}, \log \left[1 / P_{e}\left(h_{1}\right)\right] / \log \left[1 / P\left(h_{1}\right)\right]$, because $h_{1}$ and $h_{2}$ are equally informative and equally risky by hypothesis, i.e. $P\left(h_{1}\right)=$ $P\left(h_{2}\right)$ and $P_{e}\left(h_{1}\right)=P_{e}\left(h_{2}\right)$. Since their risk ratios are the same, their epistemic values are the same. 
distinguishing two kinds of justification for accepting $x$. When $P(x)=1$ and thus the epistemic value is undefined, we have trivial justification for accepting $x$ regardless of $y$. This is because when the prior probability $P(x)$ is one, the posterior probability $P_{y}(x)$ is also one, so that we take no risk at all by accepting $x$ regardless of the evidence $y$.

Of course, when we accept $x$ whose prior probability is one, we add no new information to the body of accepted propositions. So, trivial justification only allows an analytic expansion of the body of accepted propositions. Meanwhile, $J(x, y)=1-\log$ $\left[1 / P_{y}(x)\right] / \log [1 / P(x)] \geq t$ is the condition of non-trivial justification for accepting $x$ given $y$, and non-trivial justification allows a synthetic expansion of the body of accepted propositions, where new information is added. This is because $\log \left[1 / P_{y}(x)\right] / \log [1 / P(x)]$ $\geq t$ shows that the epistemic value of $x$ is sufficiently high (its risk ratio is sufficiently low). Our main focus is synthetic expansion by non-trivial justification, but we return to analytic expansion in the next section.

We can now examine how the measure $J$ of epistemic value works in the cases we discussed earlier. We begin with the conjunction fallacy in the Linda case. ${ }^{21}$ Clearly, the conjunct $q$ that Linda is a bank teller is more probable than the conjunction $p$ that Linda is a bank teller and is active in the feminist movement. So, it is less risky to accept $q$ than to accept $p$. However, the conjunction $p$ is more informative than the sole conjunct $q$. Since risk and informativeness pull us in the opposite directions, we use the $J$ measure to compare the epistemic values of the two propositions. The following assessment is based on the same probabilistic assumptions used in the confirmation analysis in Section 2. To start with the conjunction $p$, the information $i$ raises its probability, so that $P_{i}(p)>P(p)$. It

\footnotetext{
${ }^{21}$ See Shogenji (2012) for a detailed analysis of the conjunction fallacy based on the dual component model of justification.
} 
follows that $1 / P_{i}(p)<1 / P(p)$, and hence $\log \left[1 / P_{i}(p)\right]<\log [1 / P(p)]$. It follows further that $\log \left[1 / P_{i}(p)\right] / \log [1 / P(p)]<1$, so that $J(p, i)=1-\log \left[1 / P_{i}(p)\right] / \log [1 / P(p)]>0$. Meanwhile, the information $i$ lowers the probability of the conjunct $q$, so that $P_{i}(q)<P(q)$. It follows that $1 / P_{i}(q)>1 / P(q)$, and hence $\log \left[1 / P_{i}(q)\right]>\log [1 / P(q)]$. It follows further that $\log \left[1 / P_{i}(q)\right] / \log [1 / P(q)]>1$, so that $J(q, i)=1-\log \left[1 / P_{i}(q)\right] / \log [1 / P(q)]<0$. The balance is then clearly in favor of the conjunction $p$, or $J(p, i)>J(q, i)$ : the epistemic value of $p$ is higher than the epistemic value of $q$. This result is consistent with our intuitive judgment. Set aside the probability for a moment and ask yourself which of the two propositions, $p$ and $q$, you are more inclined to accept given the information $i$. If you are like the many participants in the Linda experiment, you are more inclined to accept $p$ than you are to accept $q$. This is because — we submit— the epistemic value of $p$ is higher than that of $q$. The greater informativeness of $q$ outweighs its greater risk in the case.

We want to make it clear here that we are not suggesting the many participants in the original Linda experiment were correct. It is incorrect to say that $p$ is more probable than $q$. We are suggesting that those participants mistakenly attributed their stronger inclination to accept $p$, which is actually due to the higher epistemic value of $p$, to a higher probability of $p$, confusing the epistemic value with the probability. We may call it the Lockean fallacy. The advantage of this analysis, vis-à-vis the confirmation analysis discussed in Section 2, is that it explains the fallacy from our sound inclination to accept the proposition with a higher epistemic value. The error occurs when we interpret the sound inclination in theoretical terms of probability. In contrast, the confirmation analysis leaves it unexplained why we confuse a change in probability with probability per se. 
We now return to the skeptical paradox, where the setting closely resembles the Linda case. We already know from the entailment $h \longmapsto \neg d$ that $P_{e}(h) \leq P_{e}(\neg d)$. So, accepting $\neg d$ is no riskier than accepting $h$. However, $h$ is very specific and thus much more informative than $\neg d$. Since risk and informativeness pull us in the opposite directions, we use the $J$ measure to compare the epistemic values of the two propositions. To start with $h$, the evidence $e$ - the apparent perception of a hand—raises the probability of $h$. It follows from $P_{e}(h)>P(h)$ that $J(h, e)=1-\log \left[1 / P_{e}(h)\right] / \log [1 / P(h)]>0$. The inequality only provides a lower bound of epistemic value, but it is consistent with most people's intuitive judgment that we have justification for accepting $h$ given $e$. Meanwhile, the evidence $e$ lowers the probability of $\neg d$. This is because $e$ raises the probability of $d$, as we discussed in Section 2. So, $P_{e}(\neg d)<P(\neg d)$. It follows that $J(\neg d, e)=1-$ $\log \left[1 / P_{e}(\neg d)\right] / \log [1 / P(\neg d)]<0$. Since the epistemic value of $\neg d$ is lower than that of $h$, or $J(\neg d, e)<J(h, e)$, there is no inconsistency between the two intuitions - that we have justification for accepting $h$ but we do not have justification for accepting $\neg d$.

We have examined two models of justification. The Lockean model states that we have justification for accepting $x$ when the probability $P_{y}(x)$ is sufficiently high, and that justification is closed under known entailment. Intuitions inconsistent with closure may be explained away by the pervasive probabilistic fallacy of confusing an increase in probability with a high probability. The dual component model, on the other hand, states that we have justification for accepting $x$ when its epistemic value is sufficiently highits risk ratio is sufficiently low-and that justification is not closed under known entailment. The model accommodates the first and the third intuition in the skeptical paradox, viz. once we obtain $e$, we have justification for accepting $h$, but not for 
accepting $\neg d$. It also explains that the pervasive probabilistic fallacy is not a fallacy in our epistemic practice, but a fallacy in our interpretation of a sensible epistemic practice. The explanation is this: when the epistemic value of the proposition $x$ is higher, we are more inclined to accept $x$, resulting in the sensible epistemic practice of accepting propositions with a higher epistemic value, but when we try to account for this inclination, we often mistakenly attribute it to a higher probability of $x$.

We prefer the dual component model because, other things being equal, a model that implies we have an incorrect interpretation of our sensible epistemic practice is preferable to a model that implies we regularly commit a fallacy in our epistemic practice. We are well aware that the defenders of the Lockean model would deny that other things are equal in this comparison. They would stress that justification in the Lockean model is closed under known entailment, which undergirds an expansion of the body of accepted propositions through known entailment, while justification in the dual component model is not closed under known entailment.

There is a strong and broad support for epistemic closure, especially with regard to justification (of the non-externalist kind). It is one thing to question epistemic closure with regard to knowledge since knowledge is a complex concept that is not fully understood in important respects. It is not utterly shocking if it turns out that we know $x$ but not its known logical consequence $y$ in some cases. Justification (of the nonexternalist kind) seems more straightforward and it appears unnatural and even incoherent to suggest that we have justification for accepting $x$ but not its known logical consequence $y$. 
We believe this appearance is due to conflation between closure of justification under known entailment on one hand and justifying an expansion of the body of accepted propositions through known entailment on the other. We show in the next section that we can justify an expansion of the body of accepted propositions through known entailment without closure of justification under known entailment. The expansion only requires a weaker principle of epistemic closure.

\section{Two principles of epistemic closure}

This section focuses on the second of the three intuitions that drive the skeptical paradox, viz. we have justification for expanding the body of accepted propositions through entailment. ${ }^{22}$ It seems like we have to give up the second intuition in the dual component model since justification is not closed under entailment in the model. It turns out, however, that the principle of closure under equivalence-which is weaker than the principle of closure under entailment — holds in the model and is sufficient for expansion through entailment. The following is the formal distinction of the two principles:

Closure under entailment: If $J\left(x_{1}, y\right) \geq t$ and $x_{1}-x_{2}$, then $J\left(x_{2}, y\right) \geq t$ Closure under equivalence: If $J\left(x_{1}, y\right) \geq t$ and $x_{1}|--| x_{2}$, then $J\left(x_{2}, y\right) \geq t$

" $J(x, y) \geq t$ " refers to the specific conception of justification in the dual component model, but closure under equivalence holds in any probabilistic model of justification, including the Lockean model which replaces $J(x, y) \geq t$ by $P_{y}(x) \geq t$. This is because equivalent propositions always receive the same probability — whether it is the prior probability or

\footnotetext{
${ }^{22}$ Throughout this section we assume that logical relations, such as entailment, are known.
} 
the posterior probability - in any probability distribution. As a result, any probabilistic model of justification assigns the same justificatory status to equivalent propositions.

Closure under equivalence is an extremely weak principle, but it is sufficient for expanding the body of accepted propositions through entailment. We show this in two steps. The first step justifies an expansion of the propositions we have justification for accepting in conjunction with one another through entailment. Here is the reasoning. If $x_{1}$ entails $x_{2}$, then $x_{1}$ is equivalent to the conjunction $x_{1} \wedge x_{2}$. So, by closure under equivalence, if we have justification for accepting $x_{1}$, then we have justification for accepting $x_{1} \wedge x_{2}$ - that is to say, $x_{1}$ and $x_{2}$ in conjunction with one another. Of course, it does not follow from this that we have justification for accepting $x_{2}$ on its own (unless we accept closure under entailment). The point here is that provided justification is closed under equivalence, we can expand the propositions we have justification for accepting in conjunction with one another through entailment.

The second step is to turn this result into justification for expanding the body of accepted propositions through entailment, where $x_{1}$ is already in the body of accepted propositions. There are different ways of making the second step, ${ }^{23}$ but the simplest is to argue that even after $x_{1}$ becomes part of the body of accepted propositions, $x_{1}$ is still a proposition we have justification for accepting, redundantly. If we have justification for accepting $x_{1}$, then we have justification for accepting $x_{1}$ and $x_{2}$ in conjunction with one another by the first step. There is no need to add $x_{1}$, which is already in the body of

\footnotetext{
${ }^{23}$ One possibility is to remove $x_{1}$ tentatively from the body of accepted propositions, and then evaluate $x_{1}$ to judge that we have justification for accepting it. It follows by closure under equivalence that we have justification for accepting $x_{1}$ and $x_{2}$ in conjunction with one another. However, we would have to deal with the technical problem of "belief contraction" (the difficulty of removing a proposition from a web of interconnected propositions) in this approach.
} 
accepted propositions, but there is no harm. The point is that provided justification is closed under equivalence, we have justification for adding $x_{2}$ (in conjunction with $x_{1}$ ) thereby expanding the body of accepted propositions through entailment.

Note that we would need a similar step in the Lockean model as well. In the Lockean model where justification is closed under entailment, if we have justification for accepting $x_{1}$, then we have justification for accepting $x_{2}$. So, we have justification for expanding the propositions we have justification for accepting (not necessarily in conjunction with one another) through entailment. However, this is not quite the same as expanding the body of accepted propositions through entailment, where $x_{1}$ is already in the body of accepted propositions. Of course, the proponents of the Lockean model can make the same argument we just did, viz. even after $x_{1}$ becomes part of the body of accepted propositions, $x_{1}$ is still a proposition we have justification for accepting. If we still have justification for accepting $x_{1}$, then we have justification for accepting $x_{2}$ by closure under entailment, and this allows an expansion through entailment.

There may be some objections to the second step. It may be suggested, for example, that once a proposition is accepted, it is no longer a proposition we have justification for accepting. If that is the case, we may not have justification for expanding the body of accepted propositions even if we have justification for expanding the propositions we have justification for accepting in conjunction with one another. It may also be suggested that we want the stronger principle that allows an expansion of the body of accepted propositions even if some of the propositions in the body have been accepted by an error, whereas the second step above allows an expansion only if we had justification for accepting those propositions that are already in the body. 
Whatever the merits of these objections, the proponents of closure under entailment cannot raise them against expansion by closure under equivalence at no cost. As noted already, an additional step for justifying an expansion of the body of accepted propositions is needed even if we adopt the principle of closure under entailment. So, the proponents of closure under entailment must deal with the same objections. Of course, it is open to the proponents of the Lockean model to raise the objections against the dual component model if they can provide direct justification for expanding the body of accepted propositions without relying on closure under entailment. It is unlikely, however, that any such direct justification is available only in the Lockean model without being also available in the dual component model.

Consider the following attempt at direct justification for expanding the body of accepted propositions in the Lockean model. When we come to actually accept $x_{1}$, we should consider it part of the background information $b$, so that for any $x_{2}$ entailed by $x_{1}$, $P_{b \wedge y}\left(x_{2}\right)=1$ from $x_{1} \in b$ and $x_{1} \longmapsto x_{2}$, and it follows trivially that we have justification for accepting $x_{2}$ in the Lockean model since $P_{b \wedge y}\left(x_{2}\right)=1 \geq t$. The argument seems to work, but the proponents of the dual component model can make the same move. Suppose $x_{1}$ is already part of the background information $b$, as suggested in the argument, so that $P_{b}\left(x_{2}\right)$ $=1$ from $x_{1} \in b$ and $x_{1} \vdash x_{2}$. There is then no gain in information from $x_{2}$ beyond the background information $b$ because $\log \left[1 / P_{b}\left(x_{2}\right)\right]=0$ from $P_{b}\left(x_{2}\right)=1$. There is no risk either because it also follows from $x_{1} \in b$ and $x_{1} \models x_{2}$ that $P_{b \wedge y}\left(x_{2}\right)=1$ and hence $\log$ $\left[1 / P_{b \wedge y}\left(x_{2}\right)\right]=0$. As a result, the epistemic value $J\left(x_{2}, y\right)=1-\log \left[1 / P_{y}\left(x_{2}\right)\right] / \log \left[1 / P\left(x_{2}\right)\right]$ is undefined. As explained in Section 4, this means that we have trivial justification for accepting $x_{2}$ by analytic expansion. 
In short whether it is via closure under equivalence or by analytic expansion, the dual component model allows us to expand the body of accepted propositions through entailment, just as the Lockean model does. This does not mean that the dual component model allows any expansion allowed by the Lockean model. Most importantly, if we have justification for accepting $x_{1}$, and $x_{1}$ entails $x_{2}$, the Lockean model allows us to accept $x_{2}$ on its own without also accepting $x_{1}$, while the dual component model does not allow that. For example, if we have justification for accepting the conjunction $p$ that Linda is a bank teller and is active in the feminist movement, then we have justification in the Lockean model for accepting the conjunct $q$ that Linda is a bank teller. This is not allowed in the dual component model (unless we accept $p$ and $q$ together). We believe this is consistent with our intuition in the Linda case. Similarly, if we have justification for accepting $h$ and thus justification for accepting the conjunction $h \& \neg d$ by closure under logical equivalence, then we have justification in the Lockean model for accepting $\neg d$, which contradicts our intuition that we do not have justification for accepting $\neg d$. The dual component model does not allow this reasoning. ${ }^{24}$

It is also instructive to see how the expansion in the dual component model works in making prediction. The practice of making prediction is an important reason why epistemologists like to think that justification is closed under entailment. Suppose we have justification for accepting that all emeralds are green. Can we then make a justified

\footnotetext{
${ }^{24}$ We need to be careful about the ambiguity of "we have justification for accepting $h$ and $\neg d$ " when we consult our intuition. The expression often means that we have justification for accepting $h$ and accepting $\neg d$, from which it follows (even in the dual component model) that we have justification for accepting $\neg d$. However, at issue here is the statement "we have justification for accepting $h$ and $\neg d$ " in the sense that we have justification for accepting the conjunction $h \& \rightarrow d$, from which it does not follow in the dual component model that we have justification for accepting $\neg d$.
} 
prediction that the next emerald we observe is green? There are two questions to distinguish here. One is whether we have justification for accepting that the next emerald we observe is green. We have argued that we may not: justification is not closed under entailment. The second question is whether we have justification for accepting that all emeralds are green and the next emerald we observe is green. This holds trivially because the conjunction is logically equivalent to the first conjunct, and by hypothesis we have justification for accepting the first conjunct.

This is in line with our actual practice of prediction. When we come to accept that the next emerald we observe is green because we have justification for accepting that all emeralds are green, we do not withhold judgment on the latter. We come to accept both the general proposition that all emeralds are green, and the specific proposition that the next emerald is green. We can do so because the conjunction of the general and the specific proposition is logically equivalent to the general proposition, and justification is closed under equivalence.

Some people may worry that this view undermines a common practice of refutation by Modus Tollens. Our view denies that if we have justification for accepting that all emeralds are green, then we have justification for accepting that the next emerald we observe is green. Consequently, we cannot conclude by Modus Tollens that we do not have justification for accepting that all emeralds are green when it turns out we do not have justification for accepting that the next emerald we observe is green. We do not find this troubling. We are suggesting that we must reject this line of reasoning, which the skeptic uses to question our acceptance of $h$ by challenging $\neg d$. 
What matters is that if we have justification for accepting the next emerald is not green, then we have justification for accepting that the next emerald is not green and not all emeralds are green. This allows us to expand the body of accepted propositions: once we come to accept with justification that the next emerald we observe is not green, we have justification for adding the proposition that not all emeralds are green. This is in line with our actual practice of refutation by Modus Tollens.

\section{Conclusions}

Let us recap what we have uncovered. We considered two novel Bayesian responses to the skeptical paradox. The first response combines the Lockean model of justification with the Bayesian analysis of a pervasive fallacy in the assessment of probabilities. The three intuitions that drive the skeptical paradox are incompatible in the Lockean model but the third intuition can be regarded as an instance of a pervasive fallacy in the assessment of probabilities. The second response to the skeptical paradox reconciles the first and the third intuition by the dual component model of justification, and combines it with a new analysis of epistemic closure. Although the principle of closure under entailment does not hold in the dual component model, the weaker principle of closure under equivalence holds, and is sufficient for justifying an expansion of the body of accepted propositions under entailment. We prefer the second response that accommodates all three intuitions. On this account, in order to justify the acceptance of a particular proposition about the external world, we need not defeat the demon hypothesis.

The struggle with skepticism about the external world is by no means over. The skeptic can still raise many objections to particular propositions about the external world, 
such as $h$. For instance, some epistemologists (Brueckner 1994; Vogel 2004; Yalçin 1992) have considered a formulation of the skeptical argument that is based on the principle of underdetermination instead of the principle of closure. The relation between these two formulations of the skeptical argument is not straightforward. Yet some argue (Pritchard 2005a) that the underdetermination argument remains a threat even if the closure argument fails. But as we stated at the outset, it is not our goal in this paper to establish the truth of the intuition that we have justification for accepting $h$. Our limited goal is to show that the three intuitions that drive the skeptical paradox are compatible so that we can maintain that we have justification for believing $h$ without abandoning either of the two other intuitions, and we believe we have accomplished this goal.

\section{References}

Alston, W. (1996). "Belief, Acceptance, and Religious Faith," 3-27 in D. Howard-Snyder (ed.), Faith, Freedom, and Rationality: Philosophy of Religion Today. Lanham: Rowman \& Littlefield.

Atkinson, D. (2012) "Confirmation and Justification. A Commentary on Shogenji's Measure," Synthese 184: 49-61.

Bar-Hillel, Y. and Carnap, R. (1953). "Semantic Information," British Journal for the Philosophy of Science 4: 147-157.

Brueckner, A. (1994). "The Structure of the Skeptical Argument," Philosophy and Phenomenological Research 54: 827-835.

Cohen, L. J. (1992). An Essay on Belief and Acceptance. Oxford: Clarendon Press.

Cohen, S. (2002). "Basic Knowledge and the Problem of Easy Knowledge," Philosophy and Phenomenological Research 65: 309-329.

Crupi, V., Fitelson, B. \& Tentori, K. (2008). "Probability, confirmation, and the conjunction fallacy," Thinking and Reasoning 14: 182-199.

Dretske, F. (1971). “Conclusive Reasons," Australasian Journal of Philosophy 49: 1-22.

Dretske, F. (2005). "Is Knowledge Closed Under Known Entailment?" 13-26, in M. Steup and E. Sosa (eds.) Contemporary Debate in Epistemology. Oxford: Blackwell.

Fantl, J. \& McGrath, M. (2002). "Evidence, Pragmatics, and Justification," Philosophical Review 111: 67-94.

Fantl, J. \& McGrath, M. (2007). "On Pragmatic Encroachment in Epistemology," Philosophy and Phenomenological Research 75: 558-589. 
Fantl, J. \& McGrath, M. (2009). Knowledge in an Uncertain World. Oxford: Oxford University Press.

Fantl, J. \& McGrath, M. (2012). "Pragmatic Encroachment: It's Not Just About Knowledge," Episteme 9: 27-42.

Foley, R. (2009). "Belief, Degrees of Belief, and the Lockean Thesis," in F. Huber and C. Schmidt-Petri (eds.), Degrees of Belief. Dordrecht: Springer.

Fumerton, R. (1995). Metaepistemology and Skepticism. Totowa, NJ: Rowman and Littlefield.

Huber, F. (2008a). “Assessing Theories, Bayes Style,” Synthese 161: 89-118

Huber, F. (2008b). "Hempel's Logic of Confirmation,” Philosophical Studies 139: 181189.

Jenkins, C. (2007). "Entitlement and Rationality," Synthese 157: 25-45.

Kung, P. (2010). "On Having No Reason: Dogmatism and Bayesian Confirmation," Synthese 177: 1-17.

Levi, I. (1967). Gambling with Truth. Cambridge, MA: MIT Press.

Levi, I. (1980). The Enterprise of Knowledge. Cambridge, MA: MIT Press.

Levi, I. (2004). Mild Contraction: Evaluating Loss of Information Due to Loss of Belief. Oxford: Oxford University Press.

Makinson, D. C. (1965). "The paradox of the preface," Analysis 25: 205-207.

Moretti, L. and Piazza, T. (2013). "Transmission of Justification and Warrant," in E. N. Zalta(ed.), The Stanford Encyclopedia of Philosophy (Winter Edition). http://plato.stanford.edu/archives/win2013/entries/transmission-justificationwarrant/.

Nozick, R. (1981). Philosophical Explanations. Cambridge UK: Cambridge University Press.

Okasha, S. (2004). "Wright on the Transmission of Support: a Bayesian analysis," Analysis 64: 139-146.

Popper, K. (1954). "Degree of Confirmation," The British Journal for the Philosophy of Science 5" 143-149.

Popper, K. (1963). Conjectures and Refutations. London: Routledge.

Pritchard, D. (2005a). “The Structure of Sceptical Arguments," Philosophical Quarterly $55 " 37-52$.

Pritchard, D. (2005b). "Wittgenstein's On Certainty and Contemporary Anti-Scepticism," 189-224 in D. Moyal-Sharrock and W. H. Brenner (eds.) Readings of Wittgenstein's On Certainty. London: Palgrave Macmillian.

Pryor, J. (2013). "Problems for Credulism," 89-131 in C. Tucker (ed.), Seemings and Justification: New Essays on Dogmatism and Phenomenal Conservatism. New York: Oxford University Press.

Shannon, C. and Weaver, W. (1949). The Mathematical Theory of Communication. Urbana: University of Illinois Press.

Shogenji, T. (2012). "The Degree of Epistemic Justification and the Conjunction Fallacy," Synthese 184: 29-48.

Sides, A. Osherson, D., Bonini, N. and Viale, R. (2002). "On the Reality of the Conjunction Fallacy," Memory \& Cognition 30: 191-98.

Tversky, A. and Kahneman, D. (1983). "Extensional Versus Intuitive Reasoning: The Conjunction Fallacy in Probability Judgment," Psychological Review 90: 293-315. 
Van Cleve, J. (2003). "Is Knowledge Easy or Impossible? Externalism as the Only Answer to Skepticism," in S. Luper (ed.), The Skeptics. Aldershot, UK: Ashgate.

Vogel, J. (2000). "Reliabilism Leveled," Journal of Philosophy 97: 602-23.

Vogel, J. (2004). "Skeptical Arguments,” Philosophical Issues 14: 426-55.

Vogel, J. (2008). "Epistemic Bootstrapping," Journal of Philosophy 105: 518-39.

Weatherson, B. (2007). "The Bayesian and the Dogmatist," Proceedings of the Aristotelian Society 107: 169-85.

White, R. (2006). "Problems for dogmatism," Philosophical Studies 131: 525-57.

Williamson, T. (2000). Knowledge and its Limits. Oxford: Oxford University Press.

Wright, C. (2002). "(Anti)-Sceptics Simple and Subtle: Moore and McDowell," Philosophy and Phenomenological Research 65: 330-48.

Wright, C. (2004). "Warrant for Nothing (and Foundations for Free?)," Proceedings of the Aristotelian Society Supplementary Volume 78: 167-212.

Wright, C. (2007). "Perils of Dogmatism," in S. Nucciatelli (ed.), Themes from E. G. Moore: New Essays in Epistemology and Ethics. Oxford: Oxford University Press.

Wright, C. (2014). "On Epistemic Entitlement (II): Welfare State Epistemology," 231-42 in E. Zardini and D. Dodd (eds.), Scepticism and Perceptual Justification. New York: Oxford University Press.

Yalçin, Ü. (1992). "Sceptical Arguments from Underdetermination," Philosophical Studies 68: 1-34. 